Corresponding author: Elaine. Mardis@nationwidechildrens.org

(c) 2018 Miller et al. This article is distributed under the terms of the Creative Commons Attribution License, which permits unrestricted reuse and redistribution provided that the original author and source are credited.

Ontology term: glioma

Published by Cold Spring Harbor Laboratory Press

doi: $10.1101 / \mathrm{mcs} . a 002618$

\section{Genome sequencing identifies somatic BRAF duplication c.1794_1796dupTAC;p.Thr599dup in pediatric patient with low-grade ganglioglioma}

\author{
Katherine E. Miller, ${ }^{1}$ Benjamin Kelly, ${ }_{1}$ James Fitch, ${ }^{1}$ Nicole Ross, ${ }^{1}$ \\ Matthew R. Avenarius, ${ }^{1}$ Elizabeth Varga, ${ }^{1,2}$ Daniel C. Koboldt, ${ }^{1,3}$ Daniel R. Boué, ${ }^{4}$ \\ Vincent Magrini, ${ }^{1,3}$ Scott L. Coven, ${ }^{2,3}$ Jonathan L. Finlay, ${ }^{2,3}$ Catherine E. Cottrell, ${ }^{1}$ \\ Peter White, ${ }^{1,3}$ Julie M. Gastier-Foster, ${ }^{1}$ Richard K. Wilson, ${ }^{1,3}$ Jeffrey Leonard, ${ }^{3,5}$ \\ and Elaine R. Mardis ${ }^{1,3}$

\begin{abstract}
${ }^{1}$ Institute for Genomic Medicine, Nationwide Children's Hospital, Columbus, Ohio 43205, USA; ${ }^{2}$ Division of Hematology/Oncology/Bone Marrow Transplantation, Nationwide Children's Hospital, Columbus, Ohio 43205, USA; ${ }^{3}$ Department of Pediatrics, The Ohio State University College of Medicine, Columbus, Ohio 43210, USA; ${ }^{4}$ Department of Pathology and Laboratory Medicine, Nationwide Children's Hospital, Columbus, Ohio 43205, USA; ${ }^{5}$ Department of Neurosurgery, Nationwide Children's Hospital, Columbus, Ohio 43205, USA
\end{abstract}

\begin{abstract}
Gangliogliomas (WHO grade I) are rare tumors affecting the central nervous system and are most frequently observed in children. Next-generation sequencing of tumors is being utilized at an increasing rate in both research and clinical settings to characterize the genetic factors that drive tumorigenesis. Here, we report a rare BRAF somatic mutation (NM_004333.4:c.1794_1796dupTAC; p.Thr599dup) in the tumor genome from a pediatric patient in her late teens, who was initially diagnosed with low-grade ganglioglioma at age 13. This duplication of $3 \mathrm{nt}$ introduces a second threonine residue at amino acid 599 of the BRAF protein. Based on previous studies, this variant is likely to increase kinase activity, similar to the well-characterized BRAF p.Val600Glu (V600E) pathogenic variant. In addition, although the p.T599dup somatic mutation has been documented rarely in human cancers, the variant has not been previously reported in ganglioglioma. The identification of this variant presents an opportunity to consider targeted therapy (e.g., BRAF inhibitor) for this patient.
\end{abstract}

\section{CASE PRESENTATION}

A female patient, currently $18 \mathrm{yr}$ of age, was diagnosed with a low-grade glioma at age $13 \mathrm{yr}$. She presented with blurred vision and difficulty seeing at distances. She was initially seen by an ophthalmologist who found swelling of the optic nerve and recommended an MRI of the brain, which revealed a midbrain mass extending to the medial left thalamus as well as obstructive hydrocephalus. The patient was subsequently admitted to the hospital for surgery and an endoscopic third ventriculostomy (ETV) was placed. Given the location and risk to critical structures, a biopsy of the mass was not obtained at that time; the location of the mass 
COLD SPRING HARBOR Molecular Case Studies
Somatic BRAF c.1794_1796dupTAC;p.Thr599dup in pediatric patient with ganglioglioma was consistent with a low-grade glioma. The patient did not display other symptoms prior to diagnosis (e.g., gait issues, headaches, motor weakness, vomiting, or seizures). Postoperative follow-up included a physical exam and MRI surveillance imaging every 3-4 months. During this time, imaging demonstrated slow interval growth, but the patient reported no clinical symptoms that were related to the tumor growth (e.g., headaches, vision changes). The patient was referred for chemotherapy but has not yet undergone any therapies. Four years after her initial diagnosis, a surveillance MRI indicated some central enhancement in the midbrain lesions that could represent a cyst; follow-up MRI demonstrated increased diffusion restriction and open biopsy was recommended. A needle biopsy confirmed a diagnosis of ganglioglioma, WHO grade I, and provided the specimen studied by next-generation sequencing (NGS) assays, as described herein.

\section{TECHNICAL ANALYSIS AND METHODS}

\section{Whole-Genome and Whole-Exome Sequencing}

Whole-exome sequencing (WES) and whole-genome sequencing (WGS) were performed on DNA isolated from the tumor biopsy and from peripheral blood (PBMC; i.e., normal comparator). Genomic DNA was processed for WGS using NEBNext Ultra II library prep. WES libraries were captured with the Agilent SureSelect v6 Exome kit (Agilent Technologies). Pairedend 151-bp reads were generated for exome-enriched and WGS libraries sequenced on the Illumina HiSeq 4000. Reads were aligned to the human genome reference sequence, build GRcH37, using Churchill and evaluated (Kelly et al. 2015). Sequence alignments were refined according to community-accepted guidelines for best practices (https://www. broadinstitute.org/gatk/guide/best-practices). Duplicate sequence reads were removed using samblaster-v.0.1.22, local realignment was performed on the aligned sequence data using the Genome Analysis Toolkit (GATK) (v3.7-0), and Churchill's own deterministic implementation of base quality score recalibration was used. The GATK's HaplotypeCaller was used to call germline variants. Average sequencing coverage depth for the tumor sample was 221 × (WES) and 55× (WGS); for the normal sample, coverage was 230× (WES) and $31 \times($ WGS). Somatic single-nucleotide variation (SNV) and indel detection was performed for the WGS and WES data sets separately using MuTect 2 (Cibulskis et al. 2013). Detection of copy-number variation (CNV), as well as somatic and germline structural variation (SV), was performed using WGS data.

\section{Sanger Sequencing}

DNA derived from peripheral blood and tumor was independently used as template to PCR amplify BRAF exon 15 using the following primer sequences: exon 15F 5'-GTAAAACGA CGGCCAGACTCTTCATAATGCTTGCTCTGA-3' and exon 15R 5'-CAGGAAACAGCTA TGACAGTAACTCAGCAGCATCTCAGG-3'. The resulting 251-bp products were purified (QIAGEN, 28106) and Sanger sequenced (ThermoFisher, 4336943) using M13 forward and reverse primers. Electropherograms were analyzed using Sequencher version 5.3.

\section{RNA-seq}

DNase-treated, ribo-depleted total tumor RNA was used as input for library construction using Illumina's TruSeq Stranded Total RNA Sample prep. RNA-seq data were processed using STAR-Fusion, and these data were used to detect putatively expressed gene fusions in the tumor (https://github.com/STAR-Fusion). We analyzed 125,302,701 RNA-seq reads. 
COLD SPRING HARBOR Molecular Case Studies
Somatic BRAF c.1794_1796dupTAC;p.Thr599dup in pediatric patient with ganglioglioma

\section{VARIANT INTERPRETATION}

SnpEff and custom in-house scripts were used to annotate variants (mutation and gene information), predict their functional impact on proteins, and assign population allele frequencies (Cingolani et al. 2012). Variants observed in the matched normal sample (i.e., germline) were excluded from somatic analysis. Common variants (MAF $>1 \%$ ), variants outside of coding regions ( $>4$ bp from an exon splice site), and exonic synonymous variants were also excluded from analysis. Potential variants were screened for cancer relevance based on previous reports in ClinVar, COSMIC, dbSNP, ICGC, and TCGA databases.

\section{RESULTS}

After filtering as described above, a total of 15 somatic nonsynonymous variants were further evaluated for cancer relevance. Our somatic analysis detected a pathogenic variant c.1794_1796dupTAC;p.Thr599dup in the BRAF gene (Table 1). The duplication of three nucleotides results in an in-frame introduction of a threonine residue at amino acid 599 (Fig. 1). Histology reports indicated a $70 \%$ tumor cellularity; however, the variant allele frequency (VAF) of somatic mutations suggests a much lower actual tumor cellularity. To return the results to the physician for patient care, clinical confirmation of the variant was performed by amplification of exon 15 of the BRAF gene, followed by bidirectional Sanger sequencing. Our initial confirmation assay was negative, because of low tumor content in the sample and the relative insensitivity of Sanger assays. As such, we identified a second portion of the biopsy specimen with a higher predicted tumor cellularity and isolated DNA for the Sanger confirmation, which indicated the variant was present at a low, but detectable, allelic frequency (Fig. 1). In parallel, we identified the presence of this variant and expression of the variant allele in RNA-seq data. We discovered a gene fusion transcript TFG-GPR128 in the RNA-seq data, which we determined to be irrelevant because this particular fusion has been identified in multiple healthy individuals (Chase et al. 2010). WGS analysis did not identify any $\mathrm{LOH} / \mathrm{CNV}$ regions in the tumor. Other variants (SNVs, indels, SVs) involving cancer genes were not identified in our somatic analyses. Germline WES and WGS analysis did not reveal any pathogenic variants in known cancer predisposition genes (Zhang et al. 2015).

\section{SUMMARY}

Previous in vitro studies of the p.T599dup variant demonstrated kinase activity and cellular MEK/ERK activation potential comparable to that of BRAFp.V600E, suggesting that a similar therapeutic approach as for V600E may be effective (Eisenhardt et al. 2011). Presumably, the duplication of the threonine residue destabilizes the inactive conformation of the kinase domain. The c.1794_1796TACdup variant was classified as Tier II, Level C variant in

\begin{tabular}{|c|c|c|c|c|c|c|c|}
\hline Gene & $\mathrm{Chr}$ & HGVS cDNA & $\begin{array}{l}\text { HGVS } \\
\text { protein }\end{array}$ & $\begin{array}{l}\text { Allele } \\
\text { origin }\end{array}$ & Predicted effect & $\begin{array}{l}\text { Read depth of } \\
\text { variant position }\end{array}$ & $\begin{array}{l}\text { Variant allele frequency } \\
\text { tumor } \mathrm{N} \text { reads }(\%)\end{array}$ \\
\hline$B R A F$ & $7 q 34$ & $\begin{array}{l}\text { NM_004333.4: } \\
\text { c.1794_1796dupTAC }\end{array}$ & p.Thr599dup & Somatic & $\begin{array}{l}\text { Increased kinase } \\
\text { activity (Eisenhardt } \\
\text { et al. 2011) }\end{array}$ & $\begin{array}{l}\text { WGS: } 60 \times \\
\text { WES: } 172 \times\end{array}$ & $\begin{array}{l}\text { WGS: } 5 / 60 \text { (8.3\%) } \\
\text { WES: 6/172 (3.5\%) }\end{array}$ \\
\hline
\end{tabular}

HGVS, Human Genome Variation Society; WGS, whole-genome sequencing; WES, whole-exome sequencing 


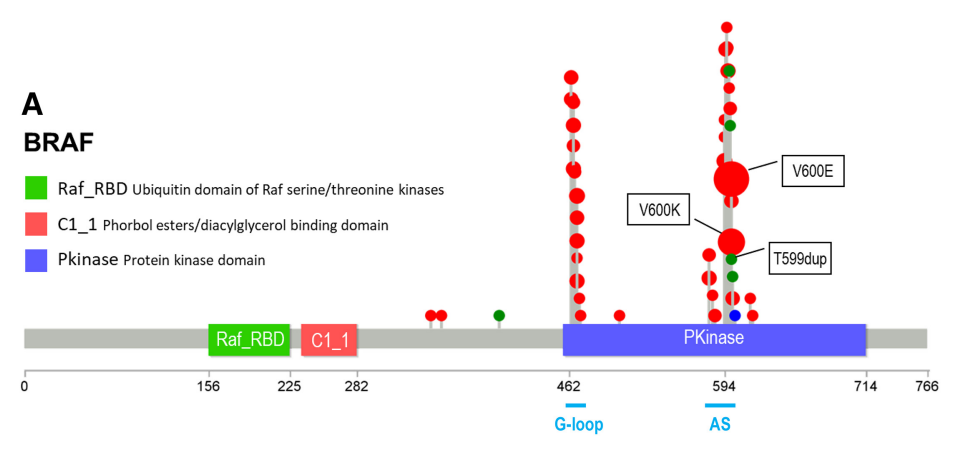

C

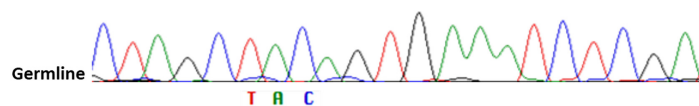

I A C

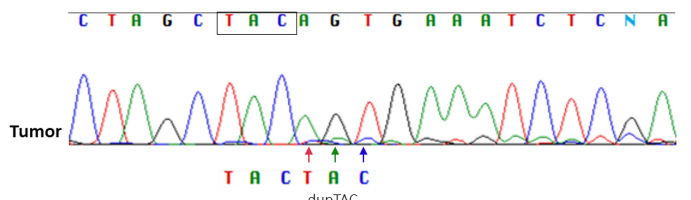

D

Schematic representation of Thr599 duplication in BRAF

$\begin{array}{cccccc}\text { L } & \text { A } & \text { T } & \text { V } & \text { K } & \\ \text { CTA } & \text { GCT } & \text { ACA } & \text { GTG } & \text { AAA } & \\ 597 & 598 & 599 & 600 & 601 & \\ \text { L } & \text { A } & \text { T } & \text { T } & \text { V } & \text { K } \\ \text { CTA } & \text { GCT } & \text { ACT } & \text { ACA } & \text { GTG } & \text { AAA } \\ 597 & 598 & 599 & \text { ^dup } & 600 & 601\end{array}$

B

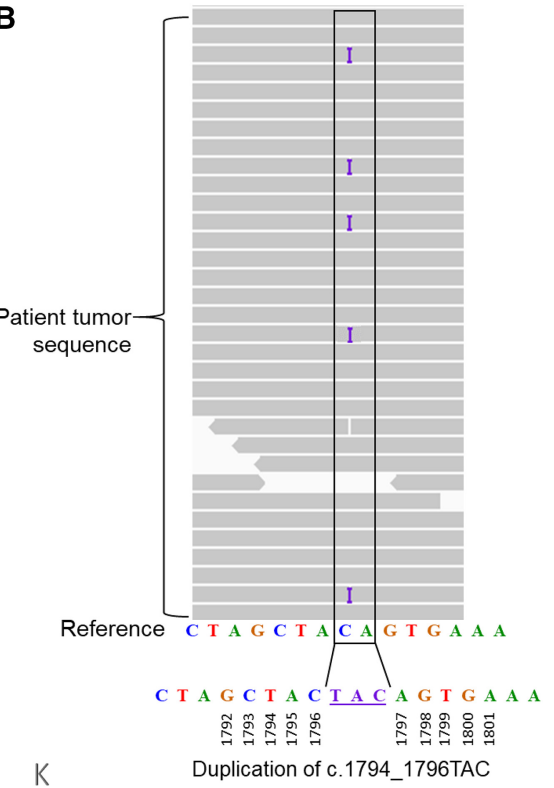

Duplication of c.1794_1796TAC

Figure 1. (A) BRAF mutations identified in tumor sequences. BRAF protein is represented here with annotated domains and amino acids (0-766) numbered underneath. Plots were generated (https://github.com/pbnjay/ lollipops) with recurrent BRAF mutations (red, missense; green, indel; blue, nonsense) identified in COSMIC (Bamford et al. 2004), with larger dots (not to scale) indicating higher-frequency mutations. Most tumor-associated BRAF variants, including the well-characterized p.V600E and p.V600K and the less frequent p.T599dup variant we report, are within the protein kinase domain, specifically in the conserved glycine motif (G-loop) or in the activation segment (AS) in exon 11 and 15, respectively. (B) Integrated Genomics Viewer (IGV) display of the c.1794_1796dupTAC. Analysis of the tumor DNA revealed a variant allele frequency of $\sim 8.3 \%$ (variant allele present in 5/60 reads). (C) Sanger sequencing of BRAF exon 15 in germline (peripheral blood) and tumor. The arrows indicate the TAC duplication, observed at low frequency, only in the tumor sample. The low peaks seen after the duplication correspond to the offset bases, because of the 3-bp duplication. (D) Schematic representation of Thr599 duplication. The specific TAC duplication is an in-frame variant, which duplicates the threonine residue at amino acid 599.

accordance with the AMP/ASCO Standards and Guidelines for the Interpretation and Reporting of Sequence Variants in Cancer (Li et al. 2017). The variant has a potential clinical actionability based on reports demonstrating therapeutic and diagnostic/prognostic utility of BRAF p.V600 substitution mutations. Although the c.1794_1796dupTAC somatic variant has been observed in other cancers, notably in thyroid cancer, melanoma, and pilocytic astrocytoma, it has not been previously described in low-grade ganglioglioma (Jones et al. 2009; Yu et al. 2009; Gauchotte et al. 2011; Menzies et al. 2012). Furthermore, the variant was not identified in germline DNA of more than 15,000 individuals in the gnomAD database (Lek et al. 2016).

We describe a somatic in-frame mutation in exon 15 of $B R A F$ in the tumor genome of a pediatric patient with ganglioglioma. The mutation duplicates the threonine residue at amino acid 599. Somatic mutations of BRAF are found with particularly high frequency in melanoma and colorectal, ovarian, and thyroid carcinomas (Davies et al. 2002; Curtin et al. 2005; Murugan et al. 2016). Specifically, the p.V600E variant accounts for $>70 \%$ of reported somatic pathogenic mutations in BRAF(Wan et al. 2004; Watson et al. 2013). Specific to gangliogliomas, BRAF substitutions are quite common. For example, BRAF p.V600E has been 
COLD SPRING HARBOR Molecular Case Studies
Somatic BRAF c.1794_1796dupTAC;p.Thr599dup in pediatric patient with ganglioglioma observed in 25\%-35\% of adult and pediatric gangliogliomas (Schindler et al. 2011; Qaddoumi et al. 2016), whereas other BRAF genetic alterations and KIAA1549-BRAF and FAM131B-BRAF fusions have also been observed in low-grade gliomas, the former fusion having been identified in gangliogliomas (Dimitriadis et al. 2013; Gupta et al. 2014; Roth et al. 2015). All of the above BRAF alterations cause increased activation of BRAF and therefore constitutive activation of the mitogen-activated protein kinase (MAPK) pathway. Previous studies have indicated that the c.1794_1796dupTAC mutation described here is pathogenic and mimics the increased oncogenic kinase activity associated with BRAF p.V600E (Eisenhardt et al. 2011). Inhibitors that can target aberrant BRAF expression (e.g., vemurafenib, dabrafenib) have been shown to positively impact disease outcomes and treatment responses in patients with BRAF V600 substitutions (Flaherty et al. 2012; Hyman et al. 2015; Karoulia et al. 2017). Specific therapeutic agents based on our patient's oncogenotype are currently being pursued.

Beyond tumor resection, radiation therapy, or chemotherapy, which often directly lead to comorbidities and/or adverse events, it is anticipated that this type of targeted therapy offers an alternative treatment strategy for this patient. It is exciting and encouraging to think about translating knowledge of somatic mutations into effective therapeutic agents, as the neurooncology community continues to reveal the molecular landscape of rare, pediatric brain tumors.

In conclusion, we suggest that screening for somatic BRAF alterations should extend beyond the well-documented hotspot variant BRAF p.V600E, as the pathogenic variant p.T599dup described here mimics the increased oncogenic kinase activity associated with p.V600E (Eisenhardt et al. 2011). It is critical that genetic assays, particularly for BRAF mutations, are capable of detecting a full range of genotypes, as the identification of pathogenic variants can lead to targeted treatment approaches for patients, as discussed here.

\section{ADDITIONAL INFORMATION}

\section{Database Deposition and Access}

The variant has been deposited in ClinVar (https://www.ncbi.nlm.nih.gov/clinvar/) under accession number SCV000611854.

\section{Ethics Statement}

This study was reviewed and approved by the Institutional Review Board (IRB) of The Research Institute at Nationwide Children's Hospital. Consent was obtained from the patient and parents for molecular genetic workup, including whole-exome and whole-genome sequencing. The protocol allowed for return of results from research sequencing studies after confirmation in a CLIA-certified laboratory.

\section{Acknowledgments}

We thank the patient and the family for their participation in our studies. We also thank the Nationwide Insurance Innovation Fund for their support of the Institute for Genomic Medicine.

\section{Author Contributions}

K.E.M. contributed to variant interpretation, initial manuscript preparation, and revision; B.K. and J.F. contributed to bioinformatics and data processing; N.R. contributed to sample processing and evaluation; M.R.A. contributed to clinical variant confirmation; E.V. and S.L.C. contributed to gathering clinical history represented in the case presentation; D.C.K. 
COLD SPRING HARBOR Molecular Case Studies
Somatic BRAF c.1794_1796dupTAC;p.Thr599dup in pediatric patient with ganglioglioma
Competing Interest Statement

The authors have declared no competing interest.

Received December 22, 2017; accepted in revised form February 6, 2018. contributed to bioinformatics and variant interpretation; D.R.B. contributed to pathology and diagnostic workup; V.M. contributed to study design; J.L.F. contributed to clinical care of patient; C.E.C. and J.M.G.-F. contributed to study design and variant interpretation; P.W. contributed to study design and bioinformatics; R.K.W. contributed to study design and initial manuscript preparation; J.L. contributed to clinical care and neurosurgery of patient; and E.R.M. contributed to study design, variant interpretation, initial manuscript preparation, and revision. All authors read and approved the final version of the manuscript.

\section{Funding}

This work was supported by the Nationwide Children's Hospital Institute for Genomic Medicine strategic fund.

\section{REFERENCES}

Bamford S, Dawson E, Forbes S, Clements J, Pettett R, Dogan A, Flanagan A, Teague J, Futreal PA, Stratton MR, et al. 2004. The COSMIC (Catalogue of Somatic Mutations in Cancer) database and website. Br J Cancer 91: 355-358.

Chase A, Ernst T, Fiebig A, Collins A, Grand F, Erben P, Reiter A, Schreiber S, Cross NC. 2010. TFG, a target of chromosome translocations in lymphoma and soft tissue tumors, fuses to GPR128 in healthy individuals. Haematologica 95: 20-26.

Cibulskis K, Lawrence MS, Carter SL, Sivachenko A, Jaffe D, Sougnez C, Gabriel S, Meyerson M, Lander ES, Getz G. 2013. Sensitive detection of somatic point mutations in impure and heterogeneous cancer samples. Nat Biotechnol 31: 213-219.

Cingolani P, Platts A, Wang LL, Coon M, Nguyen T, Wang L, Land SJ, Lu X, Ruden DM. 2012. A program for annotating and predicting the effects of single nucleotide polymorphisms, SnpEff: SNPs in the genome of Drosophila melanogaster strain w1118; iso-2; iso-3. Fly (Austin) 6: 80-92.

Curtin JA, Fridlyand J, Kageshita T, Patel HN, Busam KJ, Kutzner H, Cho KH, Aiba S, Bröcker EB, LeBoit PE, et al. 2005. Distinct sets of genetic alterations in melanoma. N Engl J Med 353: 2135-2147.

Davies H, Bignell GR, Cox C, Stephens P, Edkins S, Clegg S, Teague J, Woffendin H, Garnett MJ, Bottomley W, et al. 2002. Mutations of the BRAF gene in human cancer. Nature 417: 949-954.

Dimitriadis E, Alexiou GA, Tsotsou P, Simeonidi E, Stefanaki K, Patereli A, Prodromou N, Pandis N. 2013. BRAF alterations in pediatric low grade gliomas and mixed neuronal-glial tumors. J Neurooncol 113: 353-358.

Eisenhardt AE, Olbrich H, Röring M, Janzarik W, Anh TN, Cin H, Remke M, Witt H, Korshunov A, Pfister SM, et al. 2011. Functional characterization of a BRAF insertion mutant associated with pilocytic astrocytoma. Int J Cancer 129: 2297-2303.

Flaherty KT, Robert C, Hersey P, Nathan P, Garbe C, Milhem M, Demidov LV, Hassel JC, Rutkowski P, Mohr P, et al. 2012. Improved survival with MEK inhibition in BRAF-mutated melanoma. N Engl J Med 367: 107-114.

Gauchotte G, Philippe C, Lacomme S, Léotard B, Wissler MP, Allou L, Toussaint B, Klein M, Vignaud JM, Bressenot A. 2011. BRAF, p53 and SOX2 in anaplastic thyroid carcinoma: evidence for multistep carcinogenesis. Pathology 43: 447-452.

Gupta K, Orisme W, Harreld JH, Qaddoumi I, Dalton JD, Punchihewa C, Collins-Underwood R, Robertson T, Tatevossian RG, Ellison DW. 2014. Posterior fossa and spinal gangliogliomas form two distinct clinicopathologic and molecular subgroups. Acta Neuropathol Commun 2: 18.

Hyman DM, Puzanov I, Subbiah V, Faris JE, Chau I, Blay J-Y, Wolf J, Raje NS, Diamond EL, Hollebecque A, et al. 2015. Vemurafenib in multiple nonmelanoma cancers with BRAF V600 mutations. N Engl J Med 373: 726-736.

Jones DT, Kocialkowski S, Liu L, Pearson DM, Ichimura K, Collins VP. 2009. Oncogenic RAF1 rearrangement and a novel BRAF mutation as alternatives to KIAA1549:BRAF fusion in activating the MAPK pathway in pilocytic astrocytoma. Oncogene 28: 2119-2123.

Karoulia Z, Gavathiotis E, Poulikakos PI. 2017. New perspectives for targeting RAF kinase in human cancer. Nat Rev Cancer 17: 676-691.

Kelly BJ, Fitch JR, Hu Y, Corsmeier DJ, Zhong H, Wetzel AN, Nordquist RD, Newsom DL, White P. 2015. Churchill: an ultra-fast, deterministic, highly scalable and balanced parallelization strategy for the discovery of human genetic variation in clinical and population-scale genomics. Genome Biol 16: 6. 
COLD SPRING HARBOR Molecular Case Studies
Somatic BRAF c.1794_1796dupTAC;p.Thr599dup in pediatric patient with ganglioglioma

Lek M, Karczewski KJ, Minikel EV, Samocha KE, Banks E, Fennell T, O'Donnell-Luria AH, Ware JS, Hill AJ, Cummings BB, et al. 2016. Analysis of protein-coding genetic variation in 60,706 humans. Nature 536: 285-291.

Li MM, Datto M, Duncavage EJ, Kulkarni S, Lindeman NI, Roy S, Tsimberidou AM, Vnencak-Jones CL, Wolff DJ, Younes A, et al. 2017. Standards and guidelines for the interpretation and reporting of sequence variants in cancer: a joint consensus recommendation of the Association for Molecular Pathology, American Society of Clinical Oncology, and College of American Pathologists. J Mol Diagn 19: 4-23.

Menzies AM, Haydu LE, Visintin L, Carlino MS, Howle JR, Thompson JF, Kefford RF, Scolyer RA, Long GV. 2012. Distinguishing clinicopathologic features of patients with V600E and V600K BRAF-mutant metastatic melanoma. Clin Cancer Res 18: 3242-3249.

Murugan AK, Qasem E, Al-Hindi H, Shi Y, Alzahrani AS. 2016. Classical V600E and other non-hotspot BRAF mutations in adult differentiated thyroid cancer. J Transl Med 14: 204.

Qaddoumi I, Orisme W, Wen J, Santiago T, Gupta K, Dalton JD, Tang B, Haupfear K, Punchihewa C, Easton J, et al. 2016. Genetic alterations in uncommon low-grade neuroepithelial tumors: BRAF, FGFR1, and MYB mutations occur at high frequency and align with morphology. Acta Neuropathol 131: 833-845.

Roth JJ, Santi M, Pollock AN, Harding BN, Rorke-Adams LB, Tooke LS, Biegel JA. 2015. Chromosome band $7 q 34$ deletions resulting in KIAA 1549-BRAF and FAM 131B-BRAF fusions in pediatric low-grade gliomas. Brain Pathol 25: 182-192.

Schindler G, Capper D, Meyer J, Janzarik W, Omran H, Herold-Mende C, Schmieder K, Wesseling P, Mawrin C, Hasselblatt $M$, et al. 2011. Analysis of BRAFV600E mutation in 1,320 nervous system tumors reveals high mutation frequencies in pleomorphic xanthoastrocytoma, ganglioglioma and extra-cerebellar pilocytic astrocytoma. Acta Neuropathol 121: 397-405.

Wan PT, Garnett MJ, Roe SM, Lee S, Niculescu-Duvaz D, Good VM, Jones CM, Marshall CJ, Springer CJ, Barford D, et al. 2004. Mechanism of activation of the RAF-ERK signaling pathway by oncogenic mutations of B-RAF. Cell 116: 855-867.

Watson IR, Takahashi K, Futreal PA, Chin L. 2013. Emerging patterns of somatic mutations in cancer. Nat Rev Genet 14: 703-718.

Yu J, Deshmukh H, Gutmann RJ, Emnett RJ, Rodriguez FJ, Watson MA, Nagarajan R, Gutmann DH. 2009. Alterations of BRAF and HIPK2 loci predominate in sporadic pilocytic astrocytoma. Neurology 73: 1526-1531.

Zhang J, Walsh MF, Wu G, Edmonson MN, Gruber TA, Easton J, Hedges D, Ma X, Zhou X, Yergeau DA, et al. 2015. Germline mutations in predisposition genes in pediatric cancer. N Engl J Med 373: 2336-2346. 


\section{COLD SPRING HARBOR Molecular Case Studies}

\section{Genome sequencing identifies somatic BRAF duplication c.1794_1796dupTAC;p.Thr599dup in pediatric patient with low-grāde ganglioglioma}

Katherine E. Miller, Benjamin Kelly, James Fitch, et al.

Cold Spring Harb Mol Case Stud 2018, 4: a002618 originally published online February 6, 2018 Access the most recent version at doi: $10.1101 /$ mcs.a002618

References This article cites 26 articles, 2 of which can be accessed free at: http://molecularcasestudies.cshlp.org/content/4/2/a002618.full.html\#ref-list-1

License This article is distributed under the terms of the Creative Commons Attribution License, which permits unrestricted reuse and redistribution provided that the original author and source are credited.

Email Alerting Receive free email alerts when new articles cite this article - sign up in the box at the Service top right corner of the article or click here. 